\title{
Asymptotic Behavior of a Competition-Diffusion System with Variable Coefficients and Time Delays
}

\author{
Miguel Uh Zapata, Eric Avila Vales, and Angel G. Estrella \\ Facultad de Matemáticas, Universidad Autónoma de Yucatán, Periférico Norte, Tablaje 13615, \\ C.P. 97119, Mérida, Yucatán, Mexico
}

Correspondence should be addressed to Eric Avila Vales, avila@uady.mx

Received 26 June 2007; Accepted 15 January 2008

Recommended by Malgorzata Peszynska

A class of time-delay reaction-diffusion systems with variable coefficients which arise from the model of two competing ecological species is discussed. An asymptotic global attractor is established in terms of the variable coefficients, independent of the time delays and the effect of diffusion by the upper-lower solutions and iteration method.

Copyright (C) 2008 Miguel Uh Zapata et al. This is an open access article distributed under the Creative Commons Attribution License, which permits unrestricted use, distribution, and reproduction in any medium, provided the original work is properly cited.

\section{Introduction and main result}

The Lotka-Volterra competition model with diffusion and time delays has been the object of analysis by numerous authors under different approaches. For the case of two species, Ruan and Zhao [1] considered uniform persistence and global extinction; Lu [2] studied global attractivity, and Gourley and Ruan [3] analyzed stability and traveling fronts. The periodic case has also been considered, Feng and Wang [4] studied asymptotic stability and Zhou et al. [5] investigated the Hopf bifurcation. The cases of three and $N$-species have also been analyzed in [6-8].

In this paper, we consider the asymptotic behavior of solutions for the competitiondiffusion system with time delays of the following two species:

$$
\begin{array}{r}
\frac{\partial u_{1}}{\partial t}=A \Delta u_{1}(t, x)+u_{1}(t, x)\left[a_{1}(t, x)-b_{1}(t, x) u_{1}(t, x)-c_{1}(t, x) \int_{0}^{\infty} u_{1}(t-\tau, x) d \mu_{1}(\tau)-d_{1} u_{2}\left(t-r_{2}, x\right)\right], \\
t>0, \quad x \in \Omega, \\
\frac{\partial u_{2}}{\partial t}=A \Delta u_{2}(t, x)+u_{2}(t, x)\left[a_{2}(t, x)-b_{2}(t, x) u_{2}(t, x)-c_{2}(t, x) \int_{0}^{\infty} u_{2}(t-\tau, x) d \mu_{2}(\tau)-d_{2} u_{1}\left(t-r_{1}, x\right)\right], \\
t>0, \quad x \in \Omega,
\end{array}
$$




$$
\begin{gathered}
\frac{\partial u_{1}}{\partial \eta}=\frac{\partial u_{2}}{\partial \eta}=0, \quad t>0, x \in \partial \Omega, \\
u_{i}(t, x)=\phi_{i}(t, x) \quad(i=1,2) t \leq 0, x \in \bar{\Omega},
\end{gathered}
$$

where $\Omega \subseteq \mathbb{R}^{N}(N \geq 1)$ is a bounded domain with smooth boundary $\partial \Omega, \partial / \partial \eta$ denotes the differentiation outward normal on $\partial \Omega, A \geq 0, d_{i}>0, r_{i}>0,0<a_{i} \leq a_{i}(t, x) \leq A_{i}, 0<$ $b_{i} \leq b_{i}(t, x) \leq B_{i}, 0 \leq c_{i} \leq c_{i}(t, x) \leq C_{i}, \mu_{i}(\cdot)$ is of bounded variation with $\mu_{i}(0)=0, \phi_{i} \in$ $C^{1}((-\infty, 0]) \times \bar{\Omega}$ is bounded and nonnegative and $0 \not \equiv \phi_{i}(0, \cdot) \in C^{1}(\bar{\Omega})(i=1,2)$, and $u_{1}$ and $u_{2}$ are the density functions of two species competing for a shared limited resource.

The functions $a_{1}(x, t)$ and $a_{2}(x, t)$ denote the intrinsic growth rate of the species, $b_{1}(x, t)$ and $b_{2}(x, t)$ represent self-limitation rates, and $c_{1}(x, t)$ and $c_{2}(x, t)$ represent the coefficients of the infinite continuous delay. The constants $d_{1}, d_{2}$ represent the competition rates. The distributed time delay should be viewed as the effects of past history.

Let $M_{i}(t)$ denote the total variation of $\mu_{i}(\cdot)$ on $[0, t]$ and let $M_{i}^{ \pm}(t)=\left(M_{i}(t) \pm \mu_{i}(t)\right) / 2$, for all $t \in \mathbb{R}^{+}$. Then, $M_{i}(t)$ and $M_{i}^{ \pm}(t)$ are nonnegative and nondecreasing on $\mathbb{R}^{+}$. It easy to see that

$$
M_{i}^{+}(t)+M_{i}^{-}(t)=M_{i}(t), \quad M_{i}^{+}(t)-M_{i}^{-}(t)=\mu_{i}(t)
$$

Denote $M_{0 i}=\lim _{t \rightarrow \infty} M_{i}(t), M_{0 i}^{ \pm}=\lim _{t \rightarrow \infty} M_{i}^{ \pm}(t)$, and $\mu_{0 i}=\lim _{t \rightarrow \infty} \mu_{i}(t)$. Then,

$$
M_{0 i}^{+}+M_{0 i}^{-}=M_{0 i}, \quad M_{0 i}^{+}-M_{0 i}^{-}=\mu_{0 i} .
$$

Our result can be stated as follows.

Theorem 1.1. Assume that $b_{i}>c_{i} M_{0 i}^{-}, a_{i}-\left(C_{i} M_{0 i}^{+} A_{i}\right) /\left(b_{i}-c_{i} M_{0 i}^{-}\right)>0$, and

$$
\frac{d_{2} A_{2}\left(b_{2}-c_{2} M_{02}^{-}\right)}{\left(b_{1}-c_{1} M_{01}^{-}\right)\left[a_{2}\left(b_{2}-c_{2} M_{02}^{-}\right)-C_{2} M_{02}^{+} A_{2}\right]}<\frac{A_{2}}{A_{1}}<\frac{\left(b_{2}-c_{2} M_{02}^{-}\right)\left[a_{1}\left(b_{1}-c_{1} M_{01}^{-}\right)-C_{1} M_{01}^{+} A_{1}\right]}{d_{1} A_{1}\left(b_{1}-c_{1} M_{01}^{-}\right)} .
$$

Then, for any $\phi_{i} \in C^{1}((-\infty, 0]) \times \bar{\Omega}$ with $\phi(0, x) \not \equiv 0$, the solution of (1.1) satisfies

$$
\begin{aligned}
& 0<\alpha_{1} \leq \liminf _{t \rightarrow \infty} \min _{x \in \bar{\Omega}} u_{1}(t, x) \leq \limsup _{t \rightarrow \infty} \max _{x \in \bar{\Omega}} u_{1}(t, x) \leq \beta_{1}, \\
& 0<\alpha_{2} \leq \liminf _{t \rightarrow \infty} \min _{x \in \bar{\Omega}} u_{2}(t, x) \leq \operatorname{lim\operatorname {sup}} \max _{x \in \bar{\Omega}} u_{2}(t, x) \leq \beta_{2},
\end{aligned}
$$

where $\alpha_{1}, \alpha_{2}, \beta_{1}, \beta_{2}$ are constants given by the linear system

$$
\begin{aligned}
& a_{1}-C_{1} M_{01}^{+} \beta_{1}-d_{1} \beta_{2}-\alpha_{1}\left(B_{1}-c_{1} M_{01}^{-}\right)=0, \\
& a_{2}-C_{2} M_{02}^{+} \beta_{2}-d_{2} \beta_{1}-\alpha_{2}\left(B_{2}-c_{2} M_{02}^{-}\right)=0, \\
& A_{1}-c_{1} M_{01}^{+} \alpha_{1}-d_{1} \alpha_{2}-\beta_{1}\left(b_{1}-c_{1} M_{01}^{-}\right)=0, \\
& A_{2}-c_{2} M_{02}^{+} \alpha_{2}-d_{2} \alpha_{1}-\beta_{2}\left(b_{2}-c_{2} M_{02}^{-}\right)=0 .
\end{aligned}
$$


Miguel Uh Zapata et al.

Remark 1.2. If $\mu_{i}(\tau)=\int_{0}^{\tau} f_{i}(t) d t$ with $f_{i} \in C\left(\mathbb{R}^{+}\right) \cap L^{1}\left(\mathbb{R}^{+}\right), A=1$, and $0<a_{i}=a_{i}(t, x)=A_{i}$, $0<b_{i}=b_{i}(t, x)=B_{i}, c_{i}=c_{i}(t, x)=C_{i}=1$, then by Theorem 1.1, we get

$$
\begin{aligned}
& 0<\alpha_{1} \leq \liminf _{t \rightarrow \infty} \min _{x \in \bar{\Omega}} u_{1}(t, x) \leq \limsup _{t \rightarrow \infty} \max _{x \in \bar{\Omega}} u_{1}(t, x) \leq \beta_{1}, \\
& 0<\alpha_{2} \leq \liminf _{t \rightarrow \infty} \min _{x \in \bar{\Omega}} u_{2}(t, x) \leq \limsup _{t \rightarrow \infty} \max _{x \in \bar{\Omega}} u_{2}(t, x) \leq \beta_{2},
\end{aligned}
$$

where $\alpha_{1}, \alpha_{2}, \beta_{1}, \beta_{2}$ are constants given by the linear system

$$
\begin{aligned}
& a_{1}-M_{01}^{+} \beta_{1}-d_{1} \beta_{2}-\alpha_{1}\left(b_{1}-M_{01}^{-}\right)=0, \\
& a_{2}-M_{02}^{+} \beta_{2}-d_{2} \beta_{1}-\alpha_{2}\left(b_{2}-M_{02}^{-}\right)=0, \\
& a_{1}-M_{01}^{+} \alpha_{1}-d_{1} \alpha_{2}-\beta_{1}\left(b_{1}-M_{01}^{-}\right)=0, \\
& a_{2}-M_{02}^{+} \alpha_{2}-d_{2} \alpha_{1}-\beta_{2}\left(b_{2}-M_{02}^{-}\right)=0 .
\end{aligned}
$$

Solving this system, we have the following result:

$$
\begin{aligned}
& \alpha_{1}=\beta_{1}=\frac{a_{1}\left(b_{2}+M_{02}^{+}-M_{02}^{-}\right)-a_{2} d_{1}}{\left(b_{1}+M_{01}^{+}-M_{01}^{-}\right)\left(b_{2}+M_{02}^{+}-M_{02}^{-}\right)-d_{1} d_{2}}, \\
& \alpha_{2}=\beta_{2}=\frac{a_{2}\left(b_{1}+M_{01}^{+}-M_{01}^{-}\right)-a_{1} d_{2}}{\left(b_{1}+M_{01}^{+}-M_{01}^{-}\right)\left(b_{2}+M_{02}^{+}-M_{02}^{-}\right)-d_{1} d_{2}},
\end{aligned}
$$

which coincides with the result of [9], where the authors considered a system like (1.1) with constant coefficients.

Reaction-diffusion systems with delay have been treated by many authors. There are two ways to approach them. The first one is in the framework of semigroup theory of dynamical systems $[1,10]$. The second one is a method of upper and lower solutions, using associated monotone iterations; several authors have studied their dynamic properties $[2,9,11]$. Sometimes the birth and death rates depend on both space and time, so when we consider instantaneous and delayed interference within the species and the diffusive effects of the species, system (1.1) will be the appropriate model.

The way we organize the paper is as follows: we first introduce several results which play an important role in the proof of Theorem 1.1 which we will prove in Section 2. We will provide some numerical simulations in Section 3 in order to illustrate our theory.

The following results are developed in [11]. They considered the Volterra reactiondiffusion equations with variable coefficients:

$$
\begin{gathered}
\frac{\partial u}{\partial t}=A \Delta u(t, x)+u(t, x)\left[a(t, x)-b(t, x) u(t, x)-c(t, x) \int_{0}^{\infty} u(t-\tau, x) d \mu(\tau)\right], \quad t>0, x \in \Omega, \\
\frac{\partial u}{\partial \eta}=0, \quad t>0, x \in \partial \Omega, \\
u(t, x)=\phi(t, x), \quad t \leq 0, x \in \bar{\Omega},
\end{gathered}
$$

where $0<a_{1} \leq a(t, x) \leq A_{1}, 0<b_{1} \leq b(t, x) \leq B_{1}, 0 \leq c_{1} \leq c(t, x) \leq C_{1}$, and $\phi \in C^{1}((-\infty, 0]) \times \bar{\Omega}$ is bounded and nonnegative and $0 \not \equiv \phi(0, \cdot) \in C^{1}(\bar{\Omega})$. Let $M(t)$ denote the total variation of $\mu(\cdot)$ and define $M^{ \pm}, M_{0}^{ \pm}$of the same form as that of $M_{i}^{ \pm}, M_{0 i}^{ \pm}$, respectively. 
Lemma 1.3. Assume that $b_{1}>c_{1} M_{0}^{-}$and $a_{1}-\left(C_{1} M_{0}^{+} A_{1}\right) /\left(b_{1}-c_{1} M_{0}^{-}\right)>0$, then the solution of (1.10) satisfies

$$
\alpha \leq \liminf \min _{x \in \bar{\Omega}} u(t, x) \leq \limsup _{t \rightarrow \infty} \max _{x \in \bar{\Omega}} u(t, x) \leq \beta
$$

where

$$
\begin{aligned}
& \alpha=\frac{\left(b_{1}-c_{1} M_{0}^{-}\right) a_{1}-C_{1} M_{0}^{+} A_{1}}{\left(b_{1}-c_{1} M_{0}^{-}\right)\left(B_{1}-c_{1} M_{0}^{-}\right)-C_{1}^{2}\left(M_{0}^{+}\right)^{2}}, \\
& \beta=\frac{\left(B_{1}-c_{1} M_{0}^{-}\right) A_{1}-C_{1} M_{0}^{+} a_{1}}{\left(b_{1}-c_{1} M_{0}^{-}\right)\left(B_{1}-c_{1} M_{0}^{-}\right)-C_{1}^{2}\left(M_{0}^{+}\right)^{2}} .
\end{aligned}
$$

If $M^{+}(t)=0$, then $\mu(t)=-M^{-}(t)$. Thus,

$$
\begin{gathered}
\frac{\partial u}{\partial t}=A \Delta u(t, x)+u(t, x)\left[a(t, x)-b(t, x) u(t, x)+c(t, x) \int_{0}^{\infty} u(t-\tau, x) d M^{-}(\tau)\right], \quad t>0, x \in \Omega, \\
\frac{\partial u}{\partial \eta}=0, \quad t>0, x \in \partial \Omega \\
u(t, x)=\phi(t, x), \quad t \leq 0, x \in \bar{\Omega} .
\end{gathered}
$$

Lemma 1.4. Assume that $b_{1}>c_{1} M_{0}^{-}$and $a_{1}>0$, then the solution of (1.13) satisfies

$$
0<\alpha \leq \liminf _{t \rightarrow \infty} \min _{x \in \bar{\Omega}} u(t, x) \leq \limsup _{t \rightarrow \infty} \max _{x \in \bar{\Omega}} u(t, x) \leq \beta,
$$

where

$$
\alpha=\frac{a_{1}}{B_{1}-c_{1} M_{0}^{-}}, \quad \beta=\frac{A_{1}}{b_{1}-c_{1} M_{0}^{-}} .
$$

Lemma 1.5. If $u(t, x)$ is the solution of (1.10), then $0<u(t, x)$.

Now, we introduce the existence-comparison result for the competition-diffusion system (1.1) which is a particular case of Theorem $2.2 \mathrm{in}[12]$.

Definition 1.6. A pair of smooth functions $\tilde{u}=\left(\tilde{u}_{1}, \tilde{u}_{2}\right)$ and $\widehat{u}=\left(\widehat{u}_{1}, \widehat{u}_{2}\right)$ are called upper-lower solutions of (1.1) if $\tilde{u}_{i} \geq \widehat{u}_{i}(i=1,2)$ in $\mathbb{R} \times \bar{\Omega}$ and the following differential inequalities hold:

$$
\begin{gathered}
\frac{\partial \tilde{u}_{i}}{\partial t}-A \Delta \tilde{u}_{i} \geq \tilde{u}_{i}(t, x)\left[a_{i}(t, x)-b_{i}(t, x) \tilde{u}_{i}(t, x)-c_{i}(t, x) \int_{0}^{\infty} \tilde{u}_{i}(t-\tau, x) d \mu_{i}(\tau)-d_{i} \widehat{u}_{j}\left(t-r_{j}, x\right)\right], \\
j \neq i, t>0, x \in \Omega, \\
\frac{\partial \widehat{u}_{i}}{\partial t}-A \Delta \widehat{u}_{i} \leq \widehat{u}_{i}(t, x)\left[a_{i}(t, x)-b_{i}(t, x) \widehat{u}_{i}(t, x)-c_{i}(t, x) \int_{0}^{\infty} \widehat{u}_{i}(t-\tau, x) d \mu_{i}(\tau)-d_{i} \tilde{u}_{j}\left(t-r_{j}, x\right)\right], \\
j \neq i, t>0, x \in \Omega, \\
\frac{\partial \widehat{u}_{i}}{\partial \eta} \leq 0 \leq \frac{\partial \widetilde{u}_{i}}{\partial \eta}, \quad t>0, x \in \partial \Omega, \\
\widehat{u}_{i}(t, x) \leq \phi_{i}(t, x) \leq \tilde{u}_{i}(t, x), \quad t \leq 0, x \in \bar{\Omega}(i=1,2) .
\end{gathered}
$$


Miguel Uh Zapata et al.

Lemma 1.7. If there exists a pair of upper-lower solutions $\tilde{u}=\left(\widetilde{u}_{1}, \widetilde{u}_{2}\right)$ and $\widehat{u}=\left(\widehat{u}_{1}, \widehat{u}_{2}\right)$ of $(1.1)$, then the problem (1.1) has a unique solution $u^{*}=\left(u_{1}^{*}, u_{2}^{*}\right)$ and $\widehat{u}_{i} \leq u_{i}^{*} \leq \tilde{u}_{i}, i=1,2$.

\section{Proof of the main result}

The method of proof is via successive improvements of upper-lower solutions of suitable systems.

For given $\phi=\left(\phi_{1}, \phi_{2}\right)$ as initial conditions for the system (1.1), let $K_{1}, K_{2}$ be constants such that

$$
K_{1} \geq \max \left\{\left\|\phi_{1}\right\|, \frac{A_{1}}{b_{1}-c_{1} M_{01}^{-}}\right\}, \quad K_{2} \geq \max \left\{\left\|\phi_{2}\right\|, \frac{A_{2}}{b_{2}-c_{2} M_{02}^{-}}\right\},
$$

where $\left\|\phi_{i}\right\|=\sup \left\{\left|\phi_{i}(t, x)\right|:(t, x) \in(-\infty, 0] \times \bar{\Omega}\right\}, i=1,2$.

Then, $(0,0)$ and $\left(K_{1}, K_{2}\right)$ are a pair of lower-upper solutions of (1.1). By Lemma 1.7, there exists a unique global nonnegative solution $\left(u_{1}, u_{2}\right)$ of $(1.1)$ and it satisfies $0 \leq u_{1}(t, x) \leq K_{1}$, $0 \leq u_{2}(t, x) \leq K_{2}$.

Define $\bar{u}_{1}^{(1)}(x, t)$ and $\bar{u}_{2}^{(1)}(x, t)$ by

$$
\begin{gathered}
\frac{\partial \bar{u}_{1}^{(1)}}{\partial t}=A \Delta \bar{u}_{1}^{(1)}+\bar{u}_{1}^{(1)}\left[a_{1}(t, x)-b_{1}(t, x) \bar{u}_{1}^{(1)}+c_{1}(t, x) \int_{0}^{\infty} \bar{u}_{1}^{(1)}(t-\tau, x) d M_{1}^{-}(\tau)\right] \quad t>0, x \in \Omega, \\
\frac{\partial \bar{u}_{2}^{(1)}}{\partial t}=A \Delta \bar{u}_{2}^{(1)}+\bar{u}_{2}^{(1)}\left[a_{2}(t, x)-b_{2}(t, x) \bar{u}_{2}^{(1)}+c_{2}(t, x) \int_{0}^{\infty} \bar{u}_{2}^{(1)}(t-\tau, x) d M_{2}^{-}(\tau)\right] \quad t>0, x \in \Omega, \\
\frac{\partial \bar{u}_{1}^{(1)}}{\partial \eta}=\frac{\partial \bar{u}_{2}^{(1)}}{\partial \eta}=0, \quad t>0, x \in \partial \Omega, \\
\bar{u}_{i}^{(1)}(t, x)=K_{i}, \quad t \leq 0, x \in \bar{\Omega}(i=1,2) .
\end{gathered}
$$

Then, $(0,0)$ and $\left(\bar{u}_{1}^{(1)}, \bar{u}_{2}^{(1)}\right)$ are lower and upper solutions, and by Lemma 1.7,

$$
0 \leq u_{1} \leq \bar{u}_{1}^{(1)}, \quad 0 \leq u_{2} \leq \bar{u}_{i}^{(1)} .
$$

By Lemma 1.4, we can get

$$
0<\lim \sup _{t \rightarrow \infty} \max _{x \in \bar{\Omega}} \bar{u}_{i}^{(1)}(t, x) \leq \beta_{i}^{(0)},
$$

where

$$
\beta_{i}^{(0)}=\frac{A_{i}}{b_{i}-c_{i} M_{0 i}^{-}}, \quad i=1,2
$$

Then from (2.3) and (2.4), we get

$$
0 \leq \limsup _{t \rightarrow \infty} \max _{x \in \bar{\Omega}} u_{i}(t, x) \leq \beta_{i}^{(0)} .
$$


From (2.6) and the definition of $M_{0 i}^{+}=\lim _{t \rightarrow \infty} M_{i}^{+}(t)$, we have that for any sufficiently small $\varepsilon>0$, there exist $t_{1}^{\prime}>0$ and $t_{1}>t_{1}^{\prime}$ such that

$$
\begin{gathered}
\max _{x \in \bar{\Omega}} \bar{u}_{i}^{(1)}(t, x)<\beta_{i}^{(0)}+\varepsilon, \quad \text { for } t \geq t_{1}^{\prime}, \\
\bar{u}_{i}^{(1)}\left(t-r_{i}, x\right)<\beta_{i}^{(0)}+\varepsilon, \quad \text { for } t \geq t_{1}, \\
C_{i}\left(M_{0 i}^{+}-M_{i}^{+}\left(t-t_{1}^{\prime}\right)\right)<\varepsilon, \quad \text { for } t \geq t_{1} .
\end{gathered}
$$

Define $\underline{u}_{1}^{(1)}(t, x)$ and $\underline{u}_{2}^{(1)}(t, x)$ by

$$
\begin{gathered}
\frac{\partial \underline{u}_{1}^{(1)}}{\partial t}=A \Delta \underline{u}_{1}^{(1)}+\underline{u}_{1}^{(1)}\left[a_{1}(t, x)-b_{1}(t, x) \underline{u}_{1}^{(1)}+c_{1}(t, x) \int_{0}^{\infty} \underline{u}_{1}^{(1)}(t-\tau, x) d M_{1}^{-}(\tau)\right. \\
\left.\quad-c_{1}(t, x) \int_{0}^{\infty} \bar{u}_{1}^{(1)}(t-\tau, x) d M_{1}^{+}(\tau)-d_{1} \bar{u}_{2}^{(1)}\left(t-r_{2}, x\right)\right], \quad t>t_{1}, x \in \Omega, \\
\frac{\partial \underline{u}_{2}^{(1)}}{\partial t}=A \Delta \underline{u}_{2}^{(1)}+\underline{u}_{2}^{(1)}\left[a_{2}(t, x)-b_{2}(t, x) \underline{u}_{2}^{(1)}+c_{2}(t, x) \int_{0}^{\infty} \underline{u}_{2}^{(1)}(t-\tau, x) d M_{2}^{-}(\tau)\right. \\
\left.-c_{2}(t, x) \int_{0}^{\infty} \bar{u}_{2}^{(1)}(t-\tau, x) d M_{2}^{+}(\tau)-d_{2} \bar{u}_{1}^{(1)}\left(t-r_{1}, x\right)\right], \quad t>t_{1}, x \in \Omega, \\
\frac{\partial \underline{u}_{1}^{(1)}}{\partial \eta}=\frac{\partial \underline{u}_{2}^{(1)}}{\partial \eta}=0, \quad t>t_{1}, x \in \partial \Omega, \\
\underline{u}_{i}^{(1)}(t, x)=\frac{1}{2} u_{i}(t, x), \quad(t, x) \in\left(-\infty, t_{1}\right] \times \bar{\Omega} .
\end{gathered}
$$

Then, $\left(\underline{u}_{1}^{(1)}, \underline{u}_{2}^{(1)}\right)$ and $\left(\bar{u}_{1}^{(1)}, \bar{u}_{2}^{(1)}\right)$ are a pair of lower-upper solutions of (1.1), and by Lemma 1.7,

$$
\underline{u}_{1}^{(1)} \leq u_{1} \leq \bar{u}_{1}^{(1)}, \quad \underline{u}_{2}^{(1)} \leq u_{2} \leq \bar{u}_{2}^{(1)} .
$$

From $\bar{u}_{i}^{(1)}(t, x) \leq K_{i}$, for all $t \in \mathbb{R}$, and (2.8), for $t \geq t_{1}$, we get

$$
\begin{aligned}
& c_{i}(t, x) \int_{0}^{\infty} \bar{u}_{i}^{(1)}(t-\tau, x) d M_{i}^{+}(\tau)+d_{i} \bar{u}_{j}^{(1)}\left(t-r_{j}, x\right) \\
& \quad=c_{i}(t, x) \int_{0}^{t-t_{1}^{\prime}} \bar{u}_{i}^{(1)}(t-\tau, x) d M_{i}^{+}(\tau)+c_{i}(t, x) \int_{t-t_{1}^{\prime}}^{\infty} \bar{u}_{i}^{(1)}(t-\tau, x) d M_{i}^{+}(\tau)+d_{i} \bar{u}_{j}^{(1)}\left(t-r_{j}, x\right) \\
& \quad \leq c_{i}(t, x) \int_{0}^{t-t_{1}^{\prime}}\left(\beta_{i}^{(0)}+\varepsilon\right) d M_{i}^{+}(\tau)+c_{i}(t, x) \int_{t-t_{1}^{\prime}}^{\infty} K_{i} d M_{i}^{+}(\tau)+d_{i}\left(\beta_{j}^{(0)}+\varepsilon\right) \\
& \quad \leq C_{i}\left(M_{i}^{+}\left(t-t_{1}\right)-M(0)\right)\left(\beta_{i}^{(0)}+\varepsilon\right)+K_{i} C_{i}\left(M_{0} i^{+}-M_{i}^{+}\left(t-t_{1}\right)\right)+d_{i}\left(\beta_{j}^{(0)}+\varepsilon\right) \\
& \quad \leq C_{i} M_{0 i}^{+}\left(\beta_{i}^{(0)}+\varepsilon\right)+d_{i}\left(\beta_{j}^{(0)}+\varepsilon\right)+K_{i} \varepsilon .
\end{aligned}
$$


Miguel Uh Zapata et al.

It follows from (2.9) that, for $t>t_{1}, x \in \Omega$,

$$
\begin{aligned}
\frac{\partial \underline{u}_{1}^{(1)}}{\partial t} \geq A \Delta \underline{u}_{1}^{(1)}+\underline{u}_{1}^{(1)} & {\left[a_{1}(t, x)-b_{1}(t, x) \underline{u}_{1}^{(1)}+c_{1}(t, x) \int_{0}^{\infty} \underline{u}_{1}^{(1)}(t-\tau, x) d M_{1}^{-}(\tau)\right.} \\
& \left.-C_{1} M_{01}^{+}\left(\beta_{1}^{(0)}+\varepsilon\right)-d_{1}\left(\beta_{2}^{(0)}+\varepsilon\right)-K_{1} \varepsilon\right] \\
\frac{\partial \underline{u}_{2}^{(1)}}{\partial t} \geq A \Delta \underline{u}_{2}^{(1)}+\underline{u}_{2}^{(1)}[ & a_{2}(t, x)-b_{2}(t, x) \underline{u}_{2}^{(1)}+c_{2}(t, x) \int_{0}^{\infty} \underline{u}_{2}^{(1)}(t-\tau, x) d M_{2}^{-}(\tau) \\
& \left.-C_{2} M_{02}^{+}\left(\beta_{2}^{(0)}+\varepsilon\right)-d_{2}\left(\beta_{1}^{(0)}+\varepsilon\right)-K_{2} \varepsilon\right] .
\end{aligned}
$$

By the comparison principle, we get, for $t>t_{1}, x \in \Omega$,

$$
\underline{u}_{1}^{(1)} \geq v_{1}^{(1)}, \quad \underline{u}_{2}^{(1)} \geq v_{2}^{(1)},
$$

where $v_{1}^{(1)}$ and $v_{2}^{(1)}$ are the solutions of the following problem, respectively:

$$
\begin{gathered}
\frac{\partial v_{1}^{(1)}}{\partial t}=A \Delta v_{1}^{(1)}+v_{1}^{(1)}\left[a_{1}(t, x)-b_{1}(t, x) v_{1}^{(1)}+c_{1}(t, x) \int_{0}^{\infty} v_{1}^{(1)}(t-\tau, x) d M_{1}^{-}(\tau)\right. \\
\left.-C_{1} M_{01}^{+}\left(\beta_{1}^{(0)}+\varepsilon\right)-d_{1}\left(\beta_{2}^{(0)}+\varepsilon\right)-K_{1} \varepsilon\right], \\
\frac{\partial v_{1}^{(1)}}{\partial \eta}=0, \quad t>t_{1}, x \in \partial \Omega, \\
v_{1}^{(1)}(t, x)=\frac{1}{2} u_{1}(t, x), \quad(t, x) \in\left(-\infty, t_{1}\right] \times \bar{\Omega}, \\
\left.\frac{\partial v_{2}^{(1)}=A \Delta v_{2}^{(1)}+v_{2}^{(1)}\left[a_{2}(t, x)-b_{2}(t, x) v_{2}^{(1)}+c_{2}(t, x) \int_{0}^{\infty} v_{2}^{(1)}(t-\tau, x) d M_{2}^{-}(\tau)\right.}{\partial t}=C_{2} M_{02}^{+}\left(\beta_{2}^{(0)}+\varepsilon\right)-d_{2}\left(\beta_{1}^{(0)}+\varepsilon\right)-K_{2} \varepsilon\right], \\
\frac{\partial v_{2}^{(1)}}{\partial \eta}=0, \quad t>t_{1}, x \in \partial \Omega, \\
v_{2}^{(1)}(t, x)=\frac{1}{2} u_{2}(t, x), \quad(t, x) \in\left(-\infty, t_{1}\right] \times \bar{\Omega} .
\end{gathered}
$$

Using the three initial conditions and $\varepsilon$ sufficiently small, we have

$$
\begin{aligned}
& a_{1}-C_{1} M_{01}^{+}\left(\beta_{1}^{(0)}+\varepsilon\right)-d_{1}\left(\beta_{2}^{(0)}+\varepsilon\right)-K_{1} \varepsilon>0, \\
& a_{2}-C_{2} M_{02}^{+}\left(\beta_{2}^{(0)}+\varepsilon\right)-d_{2}\left(\beta_{1}^{(0)}+\varepsilon\right)-K_{2} \varepsilon>0 .
\end{aligned}
$$


By Lemma 1.4, we get

$$
\begin{aligned}
& 0<\frac{a_{1}-C_{1} M_{01}^{+} \beta_{1}^{(0)}-d_{1} \beta_{2}^{(0)}}{B_{1}-c_{1} M_{01}^{-}}-\varepsilon \frac{C_{1} M_{01}^{+}+d_{1}+K_{1}}{B_{1}-c_{1} M_{01}^{-}} \leq \liminf _{t \rightarrow \infty} \min _{x \in \bar{\Omega}} v_{1}^{(1)}(t, x), \\
& 0<\frac{a_{2}-C_{2} M_{02}^{+} \beta_{2}^{(0)}-d_{2} \beta_{1}^{(0)}}{B_{2}-c_{2} M_{02}^{-}}-\varepsilon \frac{C_{2} M_{02}^{+}+d_{2}+K_{2}}{B_{2}-c_{2} M_{02}^{-}} \leq \liminf _{t \rightarrow \infty} \min _{x \in \bar{\Omega}} v_{2}^{(1)}(t, x) .
\end{aligned}
$$

Then from (2.12), (2.14), and $\varepsilon$ sufficiently small, we can conclude that

$$
\begin{aligned}
& 0<\alpha_{1}^{(0)} \leq \liminf _{t \rightarrow \infty} \min _{x \in \bar{\Omega}} u_{1}(t, x) \leq \lim \sup _{t \rightarrow \infty} \max _{x \in \bar{\Omega}} u_{1}(t, x) \leq \beta_{1}^{(0)}, \\
& 0<\alpha_{2}^{(0)} \leq \liminf \min _{t \rightarrow \infty} u_{2}(t, x) \leq \operatorname{lim\operatorname {sup}} \max _{t \rightarrow \infty} u_{x \in \bar{\Omega}}(t, x) \leq \beta_{2}^{(0)},
\end{aligned}
$$

where

$$
\alpha_{1}^{(0)}=\frac{a_{1}-C_{1} M_{01}^{+} \beta_{1}^{(0)}-d_{1} \beta_{2}^{(0)}}{B_{1}-c_{1} M_{01}^{-}}, \quad \alpha_{2}^{(0)}=\frac{a_{2}-C_{2} M_{02}^{+} \beta_{2}^{(0)}-d_{2} \beta_{1}^{(0)}}{B_{2}-c_{2} M_{02}^{-}} .
$$

For any sufficiently small $\varepsilon>0$, there exist $t_{2}^{\prime}>t_{1}$ and $t_{2}>t_{2}^{\prime}$ such that

$$
\begin{array}{ll}
\min _{x \in \bar{\Omega}} \underline{u}_{i}^{(1)}(t, x)>\alpha_{i}^{(0)}-\varepsilon, & \text { for } t \geq t_{2}^{\prime}, \\
\underline{u}_{i}^{(1)}\left(t-r_{i}, x\right)>\alpha_{i}^{(0)}+\varepsilon, & \text { for } t \geq t_{2}, \\
M_{0 i}^{+}-\varepsilon<M_{i}^{+}\left(t-t_{2}^{\prime}\right), & \text { for } t \geq t_{2},
\end{array}
$$

Define $\bar{u}_{1}^{(2)}$ and $\bar{u}_{2}^{(2)}$ by

$$
\begin{gathered}
\frac{\partial \bar{u}_{1}^{(2)}}{\partial t}=A \Delta \bar{u}_{1}^{(2)}+\bar{u}_{1}^{(2)}\left[a_{1}(t, x)-b_{1}(t, x) \bar{u}_{1}^{(2)}+c_{1}(t, x) \int_{0}^{\infty} \bar{u}_{1}^{(2)}(t-\tau, x) d M_{1}^{-}(\tau)\right. \\
\left.-c_{1}(t, x) \int_{0}^{\infty} \underline{u}_{1}^{(1)}(t-\tau, x) d M_{1}^{+}(\tau)-d_{1} \underline{u}_{2}^{(1)}\left(t-r_{2}, x\right)\right], \quad t>t_{2}, x \in \Omega, \\
\frac{\partial \bar{u}_{2}^{(2)}}{\partial t}=A \Delta \bar{u}_{2}^{(2)}+\bar{u}_{2}^{(2)}\left[a_{2}(t, x)-b_{2}(t, x) \bar{u}_{2}^{(2)}+c_{2}(t, x) \int_{0}^{\infty} \bar{u}_{2}^{(2)}(t-\tau, x) d M_{2}^{-}(\tau)\right. \\
\left.-c_{2}(t, x) \int_{0}^{\infty} \underline{u}_{2}^{(1)}(t-\tau, x) d M_{2}^{+}(\tau)-d_{2} \underline{u}_{1}^{(1)}\left(t-r_{1}, x\right)\right], \quad t>t_{2}, x \in \Omega, \\
\frac{\partial \bar{u}_{1}^{(2)}}{\partial \eta}=\frac{\partial \bar{u}_{2}^{(2)}}{\partial \eta}=0, \quad t>t_{2}, x \in \partial \Omega, \\
\bar{u}_{i}^{(2)}(t, x)=K_{i}, \quad(t, x) \in\left(-\infty, t_{2}\right] \times \bar{\Omega}(i=1,2) .
\end{gathered}
$$


Then $\left(\underline{u}_{1}^{(1)}, \underline{u}_{2}^{(1)}\right)$ and $\left(\bar{u}_{1}^{(2)}, \bar{u}_{2}^{(2)}\right)$ are a pair of lower-upper solutions of (1.1). By Lemma 1.7,

$$
\underline{u}_{1}^{(1)} \leq u_{1} \leq \bar{u}_{1}^{(2)}, \quad \underline{u}_{2}^{(1)} \leq u_{2} \leq \bar{u}_{2}^{(2)} .
$$

From (2.19) and (2.20), for $t>t_{2}, x \in \Omega$, we get

$$
\begin{aligned}
\frac{\partial \bar{u}_{1}^{(2)}}{\partial t} \leq A \Delta \bar{u}_{1}^{(2)}+\bar{u}_{1}^{(2)}[ & a_{1}(t, x)-b_{1}(t, x) \bar{u}_{1}^{(2)}+c_{1}(t, x) \int_{0}^{\infty} \bar{u}_{1}^{(2)}(t-\tau, x) d M_{1}^{-}(\tau) \\
& \left.-c_{1} M_{01}^{+}\left(\alpha_{1}^{(0)}-\varepsilon\right)-d_{1}\left(\alpha_{2}^{(0)}-\varepsilon\right)+\varepsilon c_{1} \alpha_{1}^{(0)}\right] \\
\frac{\partial \bar{u}_{2}^{(2)}}{\partial t} \leq A \Delta \bar{u}_{2}^{(2)}+\bar{u}_{2}^{(2)}[ & a_{2}(t, x)-b_{2}(t, x) \bar{u}_{2}^{(2)}+c_{2}(t, x) \int_{0}^{\infty} \bar{u}_{2}^{(2)}(t-\tau, x) d M_{2}^{-}(\tau) \\
& \left.-c_{2} M_{02}^{+}\left(\alpha_{2}^{(0)}-\varepsilon\right)-d_{2}\left(\alpha_{1}^{(0)}-\varepsilon\right)+\varepsilon c_{2} \alpha_{2}^{(0)}\right] .
\end{aligned}
$$

By the comparison principle, for $t>t_{2}, x \in \Omega$, we get

$$
\bar{u}_{1}^{(2)} \leq w_{1}^{(1)}, \quad \bar{u}_{2}^{(2)} \leq w_{2}^{(1)},
$$

where $w_{1}^{(1)}$ and $w_{2}^{(1)}$ are the solutions of the following problem, respectively:

$$
\begin{gathered}
\frac{\partial w_{1}^{(1)}}{\partial t}=A \Delta w_{1}^{(1)}+w_{1}^{(1)}\left[a_{1}(t, x)-b_{1}(t, x) w_{1}^{(1)}+c_{1}(t, x) \int_{0}^{\infty} w_{1}^{(1)}(t-\tau, x) d M_{1}^{-}(\tau)\right. \\
\left.-c_{1} M_{01}^{+}\left(\alpha_{1}^{(0)}-\varepsilon\right)-d_{1}\left(\alpha_{2}^{(0)}-\varepsilon\right)+\varepsilon c_{1} \alpha_{1}^{(0)}\right], \quad t>t_{2}, x \in \Omega, \\
\frac{\partial w_{1}^{(1)}}{\partial \eta}=0, \quad t>t_{2}, x \in \partial \Omega \\
w_{1}^{(1)}(t, x)=K_{1}, \quad(t, x) \in\left(-\infty, t_{2}\right] \times \bar{\Omega}, \\
\frac{\partial w_{2}^{(1)} \partial t}{\partial t}=A \Delta w_{2}^{(1)}+w_{2}^{(1)}\left[a_{2}(t, x)-b_{2}(t, x) w_{2}^{(1)}+c_{2}(t, x) \int_{0}^{\infty} w_{2}^{(1)}(t-\tau, x) d M_{2}^{-}(\tau)\right. \\
\left.-c_{2} M_{02}^{+}\left(\alpha_{2}^{(0)}-\varepsilon\right)-d_{2}\left(\alpha_{1}^{(0)}-\varepsilon\right)+\varepsilon c_{2} \alpha_{2}^{(0)}\right], \quad t>t_{2}, x \in \Omega, \\
\frac{\partial w_{2}^{(1)}}{\partial \eta}=0, \quad t>t_{2}, x \in \partial \Omega, \\
w_{2}^{(1)}(t, x)=K_{2}, \quad(t, x) \in\left(-\infty, t_{2}\right] \times \bar{\Omega} .
\end{gathered}
$$

From (2.16), (2.18), and sufficiently small $\varepsilon$, we get

$$
\begin{aligned}
& a_{1}-c_{1} M_{01}^{+}\left(\alpha_{1}^{(0)}-\varepsilon\right)-d_{1}\left(\alpha_{2}^{(0)}-\varepsilon\right)>0, \\
& a_{2}-c_{2} M_{02}^{+}\left(\alpha_{2}^{(0)}-\varepsilon\right)-d_{2}\left(\alpha_{1}^{(0)}-\varepsilon\right)>0 .
\end{aligned}
$$


By Lemma 1.4, we get

$$
\begin{aligned}
& 0<\lim \sup _{t \rightarrow \infty} \max _{x \in \bar{\Omega}} w_{1}^{(1)}(t, x) \leq \frac{A_{1}-c_{1} M_{01}^{+} \alpha_{1}^{(0)}-d_{1} \alpha_{2}^{(0)}}{b_{1}-c_{1} M_{01}^{-}}-\varepsilon \frac{c_{1} M_{01}^{+}+d_{1}-c_{1} \alpha_{1}^{(0)}}{b_{1}-c_{1} M_{01}^{-}}, \\
& 0<\lim \sup _{t \rightarrow \infty} \max _{x \in \bar{\Omega}} w_{2}^{(1)}(t, x) \leq \frac{A_{2}-c_{2} M_{02}^{+} \alpha_{2}^{(0)}-d_{2} \alpha_{1}^{(0)}}{b_{2}-c_{2} M_{02}^{-}}-\varepsilon \frac{c_{2} M_{02}^{+}+d_{2}-c_{2} \alpha_{2}^{(0)}}{b_{2}-c_{2} M_{02}^{-}} .
\end{aligned}
$$

Then from (2.22), (2.24), and sufficiently small $\varepsilon$,

$$
\begin{aligned}
& 0<\alpha_{1}^{(0)} \leq \liminf _{t \rightarrow \infty} \min _{x \in \bar{\Omega}} u_{1}(t, x) \leq \limsup _{t \rightarrow \infty} \max _{x \in \bar{\Omega}} u_{1}(t, x) \leq \beta_{1}^{(1)}, \\
& 0<\alpha_{2}^{(0)} \leq \liminf \min _{x \rightarrow \infty} u_{2}(t, x) \leq \operatorname{lim\operatorname {sup}} \max _{x \in \bar{\Omega}} u_{2}(t, x) \leq \beta_{2}^{(1)},
\end{aligned}
$$

where

$$
\beta_{1}^{(1)}=\frac{A_{1}-c_{1} M_{01}^{+} \alpha_{1}^{(0)}-d_{1} \alpha_{2}^{(0)}}{b_{1}-c_{1} M_{01}^{-}}, \quad \beta_{2}^{(1)}=\frac{A_{2}-c_{2} M_{02}^{+} \alpha_{2}^{(0)}-d_{2} \alpha_{1}^{(0)}}{b_{2}-c_{2} M_{02}^{-}}
$$

Then,

$$
\begin{aligned}
& 0<\alpha_{1}^{(0)} \leq \beta_{1}^{(1)} \leq \beta_{1}^{(0)}, \\
& 0<\alpha_{2}^{(0)} \leq \beta_{2}^{(1)} \leq \beta_{2}^{(0)} .
\end{aligned}
$$

For any sufficiently small $\varepsilon>0$, there exist $t_{3}^{\prime}>t_{2}$ and $t_{3}>t_{3}^{\prime}$ such that

$$
\begin{gathered}
\max _{x \in \bar{\Omega}} \bar{u}_{i}^{(2)}(t, x)<\beta_{i}^{(1)}+\varepsilon, \quad \text { for } t \geq t_{3}^{\prime}, \\
\bar{u}_{i}^{(1)}\left(t-r_{i}, x\right)<\beta_{i}^{(1)}+\varepsilon, \quad \text { for } t \geq t_{3}, \\
C_{i}\left(M_{0 i}^{+}-M_{i}^{+}\left(t-t_{3}^{\prime}\right)\right)<\varepsilon, \quad \text { for } t \geq t_{3} .
\end{gathered}
$$

Define $\underline{u}_{1}^{(2)}(t, x)$ and $\underline{u}_{2}^{(2)}(t, x)$ by

$$
\begin{gathered}
\frac{\partial \underline{u}_{1}^{(2)}}{\partial t}=A \Delta \underline{u}_{1}^{(2)}+\underline{u}_{1}^{(2)}\left[a_{1}(t, x)-b_{1}(t, x) \underline{u}_{1}^{(2)}+c_{1}(t, x) \int_{0}^{\infty} \underline{u}_{1}^{(2)}(t-\tau, x) d M_{1}^{-}(\tau)\right. \\
\left.-c_{1}(t, x) \int_{0}^{\infty} \bar{u}_{1}^{(2)}(t-\tau, x) d M_{1}^{+}(\tau)-d_{1} \bar{u}_{2}^{(2)}\left(t-r_{2}, x\right)\right], \quad t>t_{3}, x \in \Omega, \\
\frac{\partial \underline{u}_{2}^{(2)}}{\partial t}=A \Delta \underline{u}_{2}^{(2)}+\underline{u}_{2}^{(2)}\left[a_{2}(t, x)-b_{2}(t, x) \underline{u}_{2}^{(2)}+c_{2}(t, x) \int_{0}^{\infty} \underline{u}_{2}^{(2)}(t-\tau, x) d M_{2}^{-}(\tau)\right. \\
\left.-c_{2}(t, x) \int_{0}^{\infty} \bar{u}_{2}^{(2)}(t-\tau, x) d M_{2}^{+}(\tau)-d_{2} \bar{u}_{1}^{(2)}\left(t-r_{1}, x\right)\right], \quad t>t_{3}, x \in \Omega, \\
\frac{\partial \underline{u}_{1}^{(2)}}{\partial \eta}=\frac{\partial \underline{u}_{2}^{(2)}}{\partial \eta}=0, \quad t>t_{3}, x \in \partial \Omega, \\
\underline{u}_{i}^{(2)}(t, x)=\frac{1}{2} u_{i}(t, x), \quad(t, x) \in\left(-\infty, t_{3}\right] \times \bar{\Omega} .
\end{gathered}
$$


Then, $\left(\underline{u}_{1}^{(2)}, \underline{u}_{2}^{(2)}\right)$ and $\left(\bar{u}_{1}^{(2)}, \bar{u}_{2}^{(2)}\right)$ are a pair of lower-upper solutions of (1.1). By Lemma 1.7,

$$
\underline{u}_{1}^{(2)} \leq u_{1} \leq \bar{u}_{1}^{(2)}, \quad \underline{u}_{2}^{(2)} \leq u_{2} \leq \bar{u}_{2}^{(2)} .
$$

From (2.30) and (2.31), for $t>t_{3}, x \in \Omega$, we get

$$
\begin{gathered}
\frac{\partial \underline{u}_{1}^{(2)} \geq A \Delta \underline{u}_{1}^{(2)}+\underline{u}_{1}^{(2)}[}{\partial t} a_{1}(t, x)-b_{1}(t, x) \underline{u}_{1}^{(2)}+c_{1}(t, x) \int_{0}^{\infty} \underline{u}_{1}^{(2)}(t-\tau, x) d M_{1}^{-}(\tau) \\
\left.-C_{1} M_{01}^{+}\left(\beta_{1}^{(1)}+\varepsilon\right)-d_{1}\left(\beta_{2}^{(1)}+\varepsilon\right)-K_{1} \varepsilon\right], \\
\frac{\partial \underline{u}_{2}^{(2)} \geq A \Delta \underline{u}_{2}^{(2)}+\underline{u}_{2}^{(2)}[}{\partial t}\left[a_{2}(t, x)-b_{2}(t, x) \underline{u}_{2}^{(2)}+c_{2}(t, x) \int_{0}^{\infty} \underline{u}_{2}^{(2)}(t-\tau, x) d M_{2}^{-}(\tau)\right. \\
\left.-C_{2} M_{02}^{+}\left(\beta_{2}^{(1)}+\varepsilon\right)-d_{2}\left(\beta_{1}^{(1)}+\varepsilon\right)-K_{2} \varepsilon\right] .
\end{gathered}
$$

By the comparison principle, for $t>t_{3}, x \in \Omega$, we get

$$
\underline{u}_{1}^{(2)} \geq v_{1}^{(2)}, \quad \underline{u}_{2}^{(2)} \geq v_{2}^{(2)},
$$

where $v_{1}^{(2)}$ and $v_{2}^{(2)}$ are the solutions of the following problem, respectively:

$$
\begin{aligned}
& \frac{\partial v_{1}^{(2)}}{\partial t}=A \Delta v_{1}^{(2)}+v_{1}^{(2)}\left[a_{1}(t, x)-b_{1}(t, x) v_{1}^{(2)}+c_{1}(t, x) \int_{0}^{\infty} v_{1}^{(2)}(t-\tau, x) d M_{1}^{-}(\tau)\right. \\
& \left.-C_{1} M_{01}^{+}\left(\beta_{1}^{(1)}+\varepsilon\right)-d_{1}\left(\beta_{2}^{(1)}+\varepsilon\right)-K_{1} \varepsilon\right], \quad t>t_{3}, x \in \Omega, \\
& \frac{\partial v_{1}^{(2)}}{\partial \eta}=0, \quad t>t_{3}, x \in \partial \Omega \\
& v_{1}^{(2)}(t, x)=\frac{1}{2} u_{1}(t, x), \quad(t, x) \in\left(-\infty, t_{3}\right] \times \bar{\Omega}, \\
& \frac{\partial v_{2}^{(2)}}{\partial t}=A \Delta v_{2}^{(2)}+v_{2}^{(2)}\left[a_{2}(t, x)-b_{2}(t, x) v_{2}^{(2)}+c_{2}(t, x) \int_{0}^{\infty} v_{2}^{(2)}(t-\tau, x) d M_{2}^{-}(\tau)\right. \\
& \left.-C_{2} M_{02}^{+}\left(\beta_{2}^{(1)}+\varepsilon\right)-d_{2}\left(\beta_{1}^{(1)}+\varepsilon\right)-K_{2} \varepsilon\right], \quad t>t_{3}, x \in \Omega, \\
& \frac{\partial v_{2}^{(2)}}{\partial \eta}=0, \quad t>t_{3}, x \in \partial \Omega \\
& v_{2}^{(2)}(t, x)=\frac{1}{2} u_{2}(t, x), \quad(t, x) \in\left(-\infty, t_{3}\right] \times \bar{\Omega} .
\end{aligned}
$$


From (2.29), (2.30), and $\varepsilon$ sufficiently small, we get

$$
\begin{aligned}
& a_{1}-C_{1} M_{01}^{+}\left(\beta_{1}^{(1)}+\varepsilon\right)-d_{1}\left(\beta_{2}^{(1)}+\varepsilon\right)-K_{1} \varepsilon>0 \\
& a_{2}-C_{2} M_{02}^{+}\left(\beta_{2}^{(1)}+\varepsilon\right)-d_{2}\left(\beta_{1}^{(1)}+\varepsilon\right)-K_{2} \varepsilon>0
\end{aligned}
$$

By Lemma 1.4 we get

$$
\begin{aligned}
& 0<\frac{a_{1}-C_{1} M_{01}^{+} \beta_{1}^{(1)}-d_{1} \beta_{2}^{(1)}}{B_{1}-c_{1} M_{01}^{-}}-\varepsilon \frac{C_{1} M_{01}^{+}+d_{1}+K_{1}}{B_{1}-c_{1} M_{01}^{-}} \leq \liminf _{t \rightarrow \infty} \min _{x \in \bar{\Omega}} v_{1}^{(1)}(t, x), \\
& 0<\frac{a_{2}-C_{2} M_{02}^{+} \beta_{2}^{(1)}-d_{2} \beta_{1}^{(1)}}{B_{2}-c_{2} M_{02}^{-}}-\varepsilon \frac{C_{2} M_{02}^{+}+d_{2}+K_{2}}{B_{2}-c_{2} M_{02}^{-}} \leq \liminf _{t \rightarrow \infty} \min _{x \in \bar{\Omega}} v_{2}^{(1)}(t, x) .
\end{aligned}
$$

From (2.33), (2.35), and for $\varepsilon$, we conclude that

$$
\begin{aligned}
& 0<\alpha_{1}^{(1)} \leq \liminf _{t \rightarrow \infty} \min _{x \in \bar{\Omega}} u_{1}(t, x) \leq \limsup _{t \rightarrow \infty} \max _{x \in \bar{\Omega}} u_{1}(t, x) \leq \beta_{1}^{(1)}, \\
& 0<\alpha_{2}^{(1)} \leq \liminf _{t \rightarrow \infty} \min _{x \in \bar{\Omega}} u_{2}(t, x) \leq \limsup _{t \rightarrow \infty} \max _{x \in \bar{\Omega}} u_{2}(t, x) \leq \beta_{2}^{(1)},
\end{aligned}
$$

where

$$
\alpha_{1}^{(1)}=\frac{a_{1}-C_{1} M_{01}^{+} \beta_{1}^{(1)}-d_{1} \beta_{2}^{(1)}}{B_{1}-c_{1} M_{01}^{-}}, \quad \alpha_{2}^{(1)}=\frac{a_{2}-C_{2} M_{02}^{+} \beta_{2}^{(1)}-d_{2} \beta_{1}^{(1)}}{B_{2}-c_{2} M_{02}^{-}}
$$

Then,

$$
\begin{aligned}
& 0<\alpha_{1}^{(0)} \leq \alpha_{1}^{(1)} \leq \beta_{1}^{(1)} \leq \beta_{1}^{(0)}, \\
& 0<\alpha_{2}^{(0)} \leq \alpha_{2}^{(1)} \leq \beta_{2}^{(1)} \leq \beta_{2}^{(0)} .
\end{aligned}
$$

Define the sequences $\alpha_{1}^{(k)}, \alpha_{2}^{(k)}, \beta_{1}^{(k)}$, and $\beta_{2}^{(k)}$ as follows:

$$
\begin{aligned}
\alpha_{1}^{(k)}=\frac{a_{1}-C_{1} M_{01}^{+} \beta_{1}^{(k)}-d_{1} \beta_{2}^{(k)}}{B_{1}-c_{1} M_{01}^{-}}, & \alpha_{2}^{(k)}=\frac{a_{2}-C_{2} M_{02}^{+} \beta_{2}^{(k)}-d_{2} \beta_{1}^{(k)}}{B_{2}-c_{2} M_{02}^{-}}, \\
\beta_{1}^{(k+1)}=\frac{A_{1}-c_{1} M_{01}^{+} \alpha_{1}^{(k)}-d_{1} \alpha_{2}^{(k)}}{b_{1}-c_{1} M_{01}^{-}}, & \beta_{2}^{(k+1)}=\frac{A_{2}-c_{2} M_{02}^{+} \alpha_{2}^{(k)}-d_{2} \alpha_{1}^{(k)}}{b_{2}-c_{2} M_{02}^{-}} \\
\beta_{1}^{(0)}=\frac{A_{1}}{b_{1}-c_{1} M_{01}^{-}}, & \beta_{2}^{(0)}=\frac{A_{2}}{b_{2}-c_{2} M_{02}^{-}} .
\end{aligned}
$$


Lemma 2.1. For the above-defined sequences, one has

$$
\left[\alpha_{1}^{(k+1)}, \beta_{1}^{(k+1)}\right] \subseteq\left[\alpha_{1}^{(k)}, \beta_{1}^{(k)}\right], \quad\left[\alpha_{2}^{(k+1)}, \beta_{2}^{(k+1)}\right] \subseteq\left[\alpha_{2}^{(k)}, \beta_{2}^{(k)}\right], \quad k \geq 0 .
$$

Proof. For $k=0$, it has been shown that $\left[\alpha_{1}^{(1)}, \beta_{1}^{(1)}\right] \subseteq\left[\alpha_{1}^{(0)}, \beta_{1}^{(0)}\right]$ and $\left[\alpha_{2}^{(1)}, \beta_{2}^{(1)}\right] \subseteq\left[\alpha_{2}^{(0)}, \beta_{2}^{(1)}\right]$. Using induction, we can complete the proof.

Lemma 2.1 implies that

$$
\lim _{k \rightarrow \infty} \alpha_{1}^{(k)}, \quad \lim _{k \rightarrow \infty} \alpha_{2}^{(k)}, \quad \lim _{k \rightarrow \infty} \beta_{1}^{(k)}, \quad \lim _{k \rightarrow \infty} \beta_{2}^{(k)}
$$

exist, denoted as $\alpha_{1}, \alpha_{2}, \beta_{1}$, and $\beta_{2}$, respectively. From (2.41), we have the following linear system by which we can obtain the numbers $\alpha_{1}, \alpha_{2}, \beta_{1}$, and $\beta_{2}$ :

$$
\begin{array}{ll}
\alpha_{1}=\frac{a_{1}-C_{1} M_{01}^{+} \beta_{1}-d_{1} \beta_{2}}{B_{1}-c_{1} M_{01}^{-}}, & \alpha_{2}=\frac{a_{2}-C_{2} M_{02}^{+} \beta_{2}-d_{2} \beta_{1}}{B_{2}-c_{2} M_{02}^{-}}, \\
\beta_{1}=\frac{A_{1}-c_{1} M_{01}^{+} \alpha_{1}-d_{1} \alpha_{2}}{b_{1}-c_{1} M_{01}^{-}}, & \beta_{2}=\frac{A_{2}-c_{2} M_{02}^{+} \alpha_{2}-d_{2} \alpha_{1}}{b_{2}-c_{2} M_{02}^{-}} .
\end{array}
$$

Lemma 2.2. For the solutions of (1.1), one has

$$
\begin{aligned}
& 0<\alpha_{1}^{(k)} \leq \liminf _{t \rightarrow \infty} \min _{x \in \bar{\Omega}} u_{1}(t, x) \leq \limsup _{t \rightarrow \infty} \max _{x \in \bar{\Omega}} u_{1}(t, x) \leq \beta_{1}^{(k)}, \quad k \geq 0, \\
& 0<\alpha_{2}^{(k)} \leq \liminf \min _{t \rightarrow \infty} u_{2}(t, x) \leq \limsup _{t \rightarrow \infty} \max _{x \in \bar{\Omega}} u_{2}(t, x) \leq \beta_{2}^{(k)}, \quad k \geq 0 .
\end{aligned}
$$

Proof. We have shown that (2.45) and (3.1) are valid for $k=0,1$. Using induction and repeating the above process, we can complete the proof.

Combining the above lemmas, we can complete the proof of Theorem 1.1

Remark 2.3. Following the same kind of proof for Theorem 1.1, it can be shown that the same conclusions hold if instead of system (1.1) we work with solutions of

$$
\begin{gathered}
\frac{\partial v_{1}}{\partial t}=A \Delta v_{1}(t, x)+v_{1}(t, x)\left[a_{1}(t, x)-b_{1}(t, x) v_{1}(t, x)-c_{1}(t, x) \int_{0}^{\infty} v_{1}(t-\tau, x) d \mu_{1}(\tau)-d_{1} v_{2}\left(t-r_{2}, x\right)\right], \\
t>0, x \in \Omega, \\
\frac{\partial v_{2}}{\partial t}=B \Delta v_{2}(t, x)+v_{2}(t, x)\left[a_{2}(t, x)-b_{2}(t, x) v_{2}(t, x)-c_{2}(t, x) \int_{0}^{\infty} v_{2}(t-\tau, x) d \mu_{2}(\tau)-d_{2} v_{1}\left(t-r_{1}, x\right)\right], \\
t>0, x \in \Omega, \\
\frac{\partial v_{1}}{\partial \eta}=\frac{\partial v_{2}}{\partial \eta}=0, \quad t>0, x \in \partial \Omega \\
v_{i}(t, x)=\phi_{i}(t, x) \quad(i=1,2) t \leq 0, x \in \bar{\Omega}
\end{gathered}
$$

as expected since they do not include the coefficients of diffusion. We thank one of the referees for his comments regarding this matter. 


\section{Numerical simulations}

In this section, we present some numerical results that agree with Theorem 1.1 proved above. We used the method of upper and lower solutions as developed by Pao $[12,13]$ discretizing the systems into finite difference systems. On both examples, the domain used is $\Omega=(0,12)$.

Example 3.1. In this example, we work with coefficients that depend on $t$ and initial values that depend on $(t, x)$.

Consider the system

$$
\begin{gathered}
\frac{\partial u_{1}}{\partial t}=\frac{\partial^{2} u_{1}}{\partial x^{2}}+u_{1}\left[(\sin (t)+2)-\left(\frac{1}{1+x^{2}}+10\right) u_{1}\right. \\
\left.\quad-(\cos (t)+1.01) \int_{0}^{\infty} \frac{1}{1+\tau^{2}} u_{1}(t-\tau, x) d \tau-\frac{1}{10} u_{2}(t-2, x)\right], \quad t \in[0, T], x \in[0,12], \\
\frac{\partial u_{2}}{\partial t}=\frac{\partial^{2} u_{2}}{\partial x^{2}}+u_{2}\left[\left(\sqrt{\sin ^{2}(t)}+1\right)-\left(\frac{x}{1+x^{2}}+7.5\right) u_{2}\right. \\
\left.-\left(\sin \left(t^{2}\right)+1.01\right) \int_{0}^{\infty} \frac{1}{1+\tau^{2}} u_{2}(t-\tau, x) d \tau-\frac{1}{2} u_{1}(t-1.5, x)\right], \quad t \in[0, T], x \in[0,12], \\
\quad \frac{\partial u_{i}}{\partial x}(0, t)=\frac{\partial u_{i}}{\partial x}(12, t)=0 \quad(i=1,2), t>0, \\
u_{1}(t, x)=\frac{1}{2} \sin \left(\frac{1}{2} t\right)+1.01, \quad t \leq 0, x \in[0,12], \\
u_{2}(t, x)=\frac{1}{2}\left(\sin \left(\frac{1}{2} t\right)+\sin \left(\frac{1}{2} x\right)\right)+1.01, \quad t \leq 0, x \in[0,12] .
\end{gathered}
$$

It is easy to see that this system satisfies the conditions of the main theorem (Theorem 1.1), therefore the global attractors are defined by the solutions of the linear system

$$
\begin{aligned}
& \alpha_{1}(11-0)=1-2.01 \frac{\pi}{2} \beta_{1}-\frac{1}{10} \beta_{2} \\
& \alpha_{2}(1.5-0)=1-2.01 \frac{\pi}{2} \beta_{2}-\beta_{1} \\
& \beta_{1}(10-0)=3-0.01 \frac{\pi}{2} \alpha_{1}-\frac{1}{10} \alpha_{2} \\
& \beta_{2}(0.5-0)=2-0.01 \frac{\pi}{2} \alpha_{2}-\alpha_{1}
\end{aligned}
$$

that is, the global attractors for $u_{1}$ and $u_{2}$ are given by $[0.002245,0.299884]$ and $[0.011192,0.285680]$, respectively. Figure 1 shows the numerical simulation of the solution of this system and on it we can notice the global attractors obtained before. 


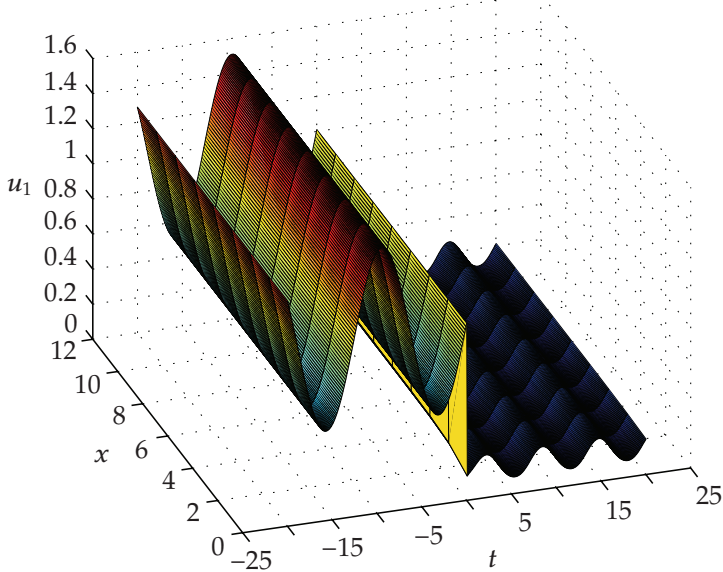

(a)

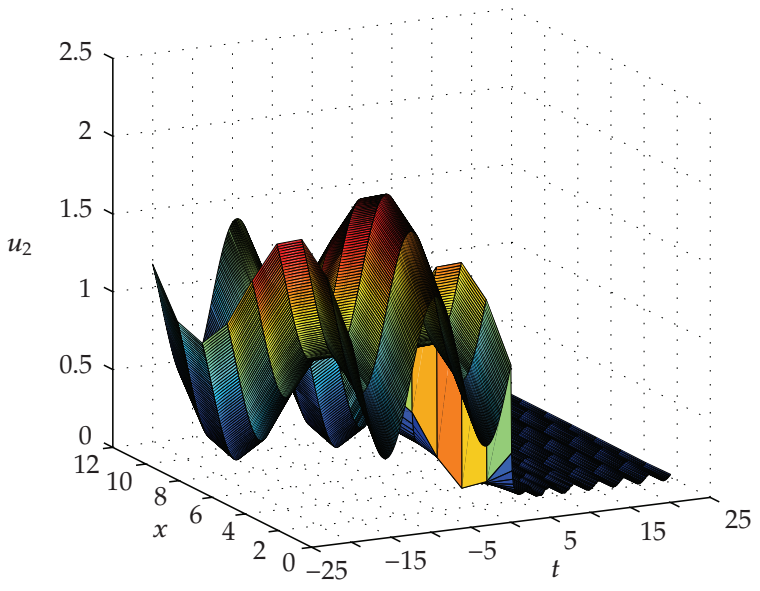

(b)

Figure 1: Numerical simulation of Example 3.1.

Example 3.2. According to the main theorem (Theorem 1.1), the global attractors depend only on the coefficients and not on the initial values. In this example, we only change the initial functions of the above example:

$$
\begin{gathered}
u_{1}(t, x)=\sin (x)+1.01, \quad t \leq 0, x \in[0,12] \\
u_{2}(t, x)=\frac{1}{200}\left(1+t^{2}\right) \sin \left(\frac{\pi x}{12}\right)+0.01, \quad t \leq 0, x \in[0,12],
\end{gathered}
$$




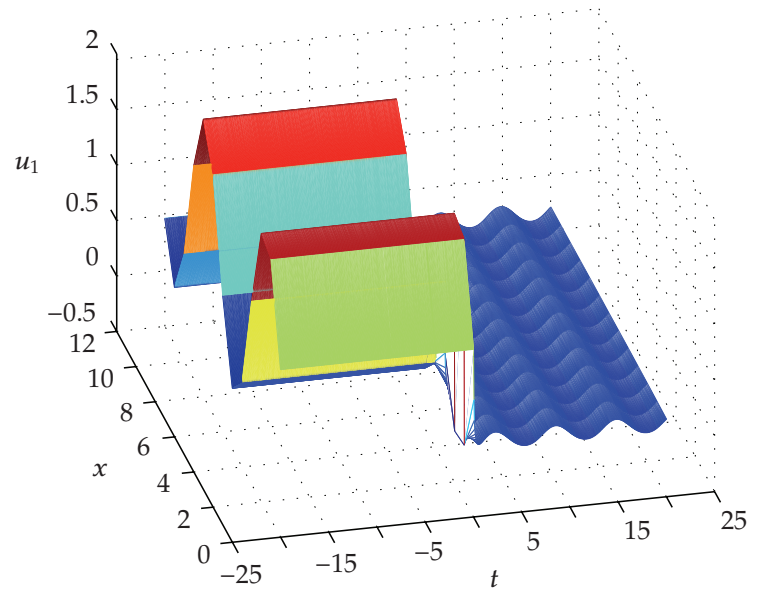

(a)

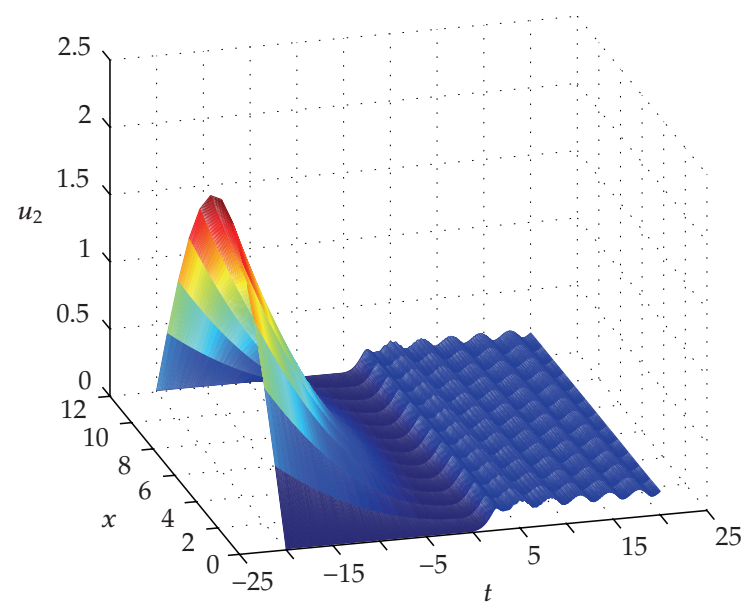

(b)

Figure 2: Numerical simulation of Example 3.2.

to obtain the same attractors as in Example 3.1. Figure 2 shows the numerical simulation of the solution of this system and we observe that they have the attractors expected.

\section{Acknowledgments}

The research of the first and second authors was supported in part by CONACYT under Grant SEP-2003-C02-44029. The research of the second author was supported in part by SNI under Grant 15284. The research of the third author was supported in part by UADY under Grant PRIORI-FMAT-04-002. 


\section{References}

[1] S. G. Ruan and X.-Q. Zhao, "Persistence and extinction in two species reaction-diffusion systems with delays," Journal of Differential Equations, vol. 156, no. 1, pp. 71-92, 1999.

[2] X. Lu, "Persistence and extinction in a competition-diffusion system with time delays," The Canadian Applied Mathematics Quarterly, vol. 2, no. 2, pp. 231-246, 1994.

[3] S. A. Gourley and S. Ruan, "Convergence and travelling fronts in functional differential equations with nonlocal terms: a competition model," SIAM Journal on Mathematical Analysis, vol. 35, no. 3, pp. 806-822, 2003.

[4] W. Feng and F. Wang, "Asymptotic periodicity and permanence in a competition-diffusion system with discrete delays," Applied Mathematics and Computation, vol. 89, no. 1-3, pp. 99-110, 1998.

[5] L. Zhou, Y. Tang, and S. Hussein, "Periodic bifurcation solution for a delay competition diffusion system," Nonlinear Analysis: Theory, Methods \& Applications, vol. 47, no. 9, pp. 6073-6084, 2001.

[6] W. Feng and X. Lu, "Harmless delays for permanence in a class of population models with diffusion effects," Journal of Mathematical Analysis and Applications, vol. 206, no. 2, pp. 547-566, 1997.

[7] C. V. Pao, "Global asymptotic stability of Lotka-Volterra 3-species reaction-diffusion systems with time delays," Journal of Mathematical Analysis and Applications, vol. 281, no. 1, pp. 186-204, 2003.

[8] C. V. Pao, "Global asymptotic stability of Lotka-Volterra competition systems with diffusion and time delays," Nonlinear Analysis: Real World Applications, vol. 5, no. 1, pp. 91-104, 2004.

[9] Y. Wang and Y. Meng, "Asymptotic behavior of a competition-diffusion system with time delays," Mathematical and Computer Modelling, vol. 38, no. 5-6, pp. 509-517, 2003.

[10] J. Wu and X.-Q. Zhao, "Diffusive monotonicity and threshold dynamics of delayed reaction diffusion equations," Journal of Differential Equations, vol. 186, no. 2, pp. 470-484, 2002.

[11] B. Shi and Y. Chen, "A prior bounds and stability of solutions for a Volterra reaction-diffusion equation with infinite delay," Nonlinear Analysis: Theory, Methods \& Applications, vol. 44, no. 1, pp. 97-121, 2001.

[12] C. V. Pao, "Systems of parabolic equations with continuous and discrete delays," Journal of Mathematical Analysis and Applications, vol. 205, no. 1, pp. 157-185, 1997.

[13] C. V. Pao, Nonlinear Parabolic and Elliptic Equations, New York, NY, USA, Plenum Press, 1992. 


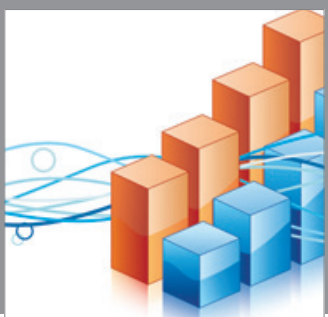

Advances in

Operations Research

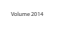

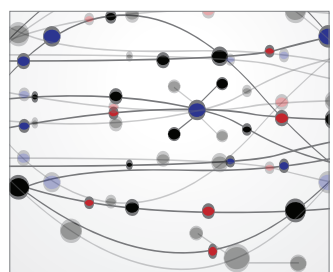

\section{The Scientific} World Journal
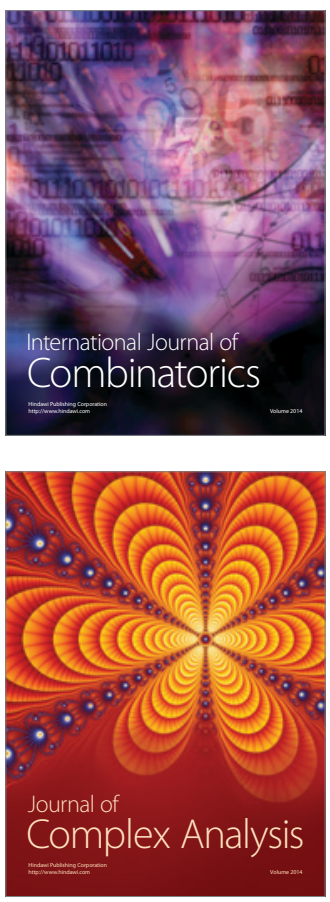

International Journal of

Mathematics and

Mathematical

Sciences
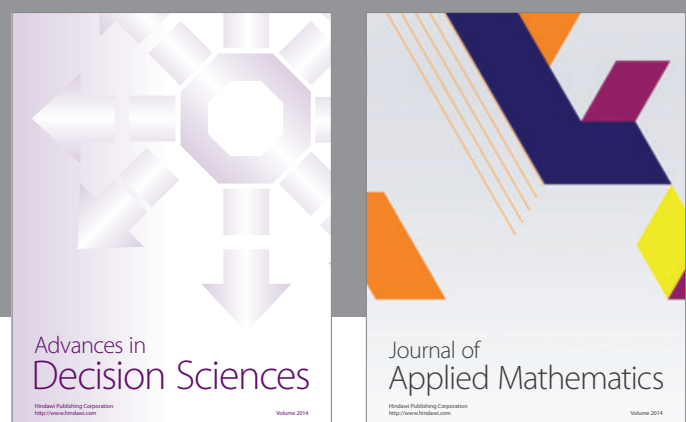

Journal of

Applied Mathematics
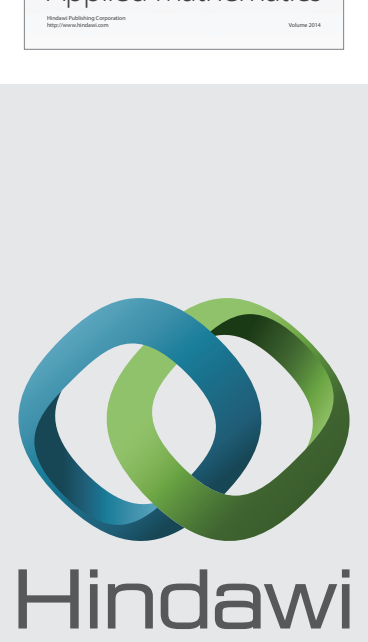

Submit your manuscripts at http://www.hindawi.com
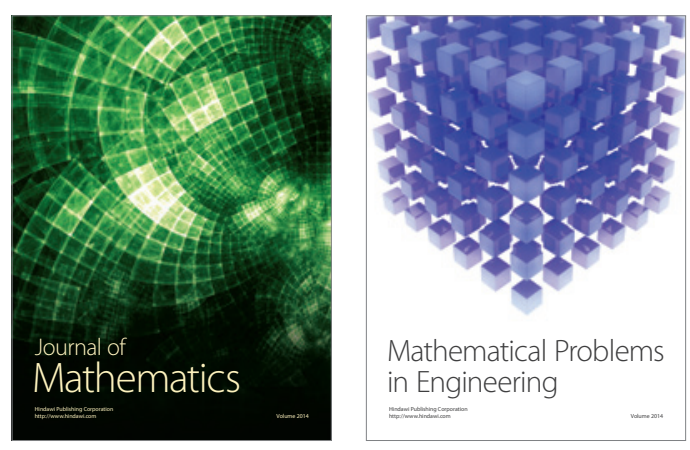

Mathematical Problems in Engineering
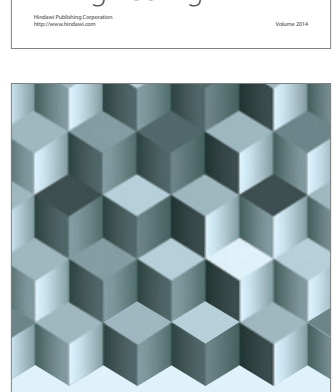

Journal of

Function Spaces
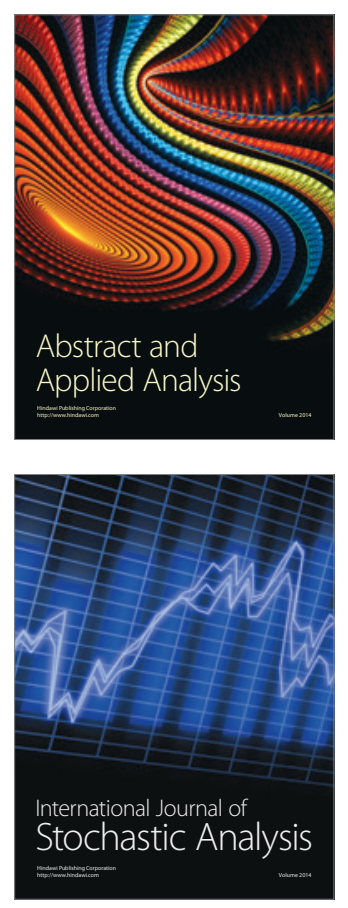

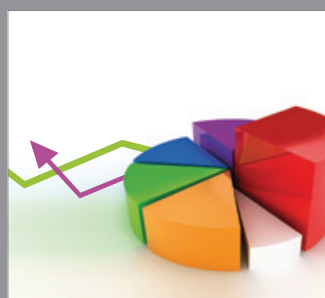

ournal of

Probability and Statistics

Promensencen
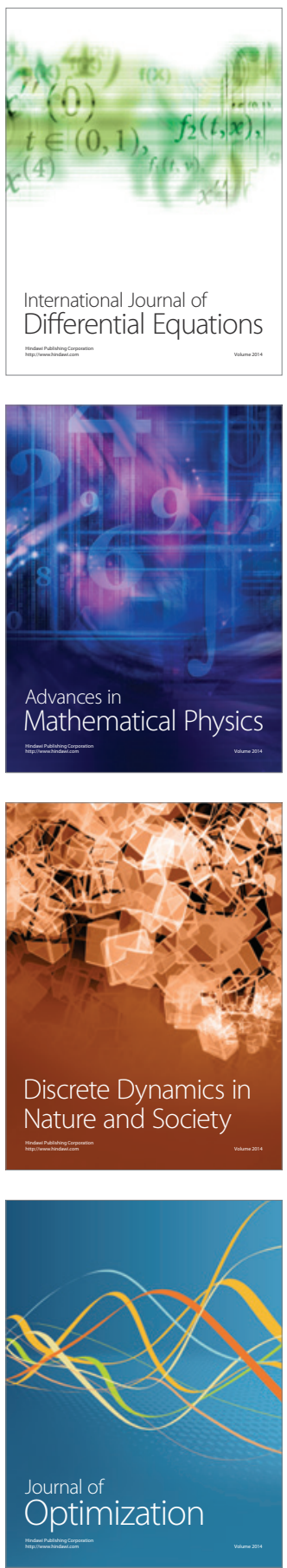